\title{
Belphégor
}

\section{Lazzarin, Stefano, Felice Italo Beneduce, Eleonora Conti, Fabrizio Foni, Rita Fresu, Claudia Zudini. Il fantastico italiano. Bilancio critico e bibliografia commentata (dal 1980 a oggi)}

\section{Vittorio Frigerio}

\section{(2) OpenEdition}

\section{Journals}

Electronic version

URL: https://journals.openedition.org/belphegor/2143

DOI: 10.4000/belphegor.2143

ISSN: 1499-7185

Publisher

LPCM

Electronic reference

Vittorio Frigerio, "Lazzarin, Stefano, Felice Italo Beneduce, Eleonora Conti, Fabrizio Foni, Rita Fresu,

Claudia Zudini. II fantastico italiano. Bilancio critico e bibliografia commentata (dal 1980 a oggi)", Belphégor [Online], 18-1 | 2020, Online since 14 January 2020, connection on 29 June 2021. URL: http:// journals.openedition.org/belphegor/2143; DOI: https://doi.org/10.4000/belphegor.2143

This text was automatically generated on 29 June 2021.

\section{(i) 89


Lazzarin, Stefano, Felice Italo Beneduce, Eleonora Conti, Fabrizio Foni, Rita Fresu, Claudia Zudini. Il fantastico italiano. Bilancio critico e bibliografia commentata (dal 1980 a oggi)

Vittorio Frigerio 


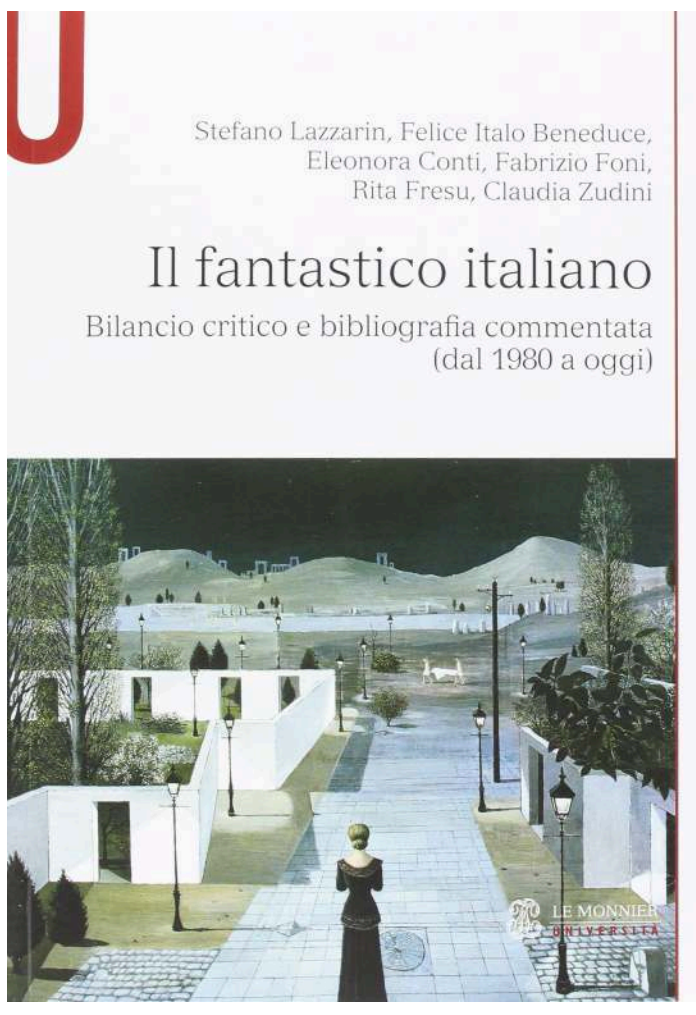

1 Il genere fantastico - ammesso e non necessariamente concesso che si possa parlare di genere a riguardo di questo fenomeno letterario straordinariamente durevole e popolare - è sempre stato fin dai suoi inizi estremamente problematico da definire. Quel miscuglio particolare di meraviglioso e reale nel quale si riscontrava l'originalità indiscussa di E.T.H Hoffman, lo scrittore tedesco il cui successo in Francia nel corso dell'ottocento lancia la "moda" del fantastico, ha come particolarità principale di restare, come gli spettri che talvolta evoca, ben difficile da afferrare, definire, ridurre o etichettare. $\mathrm{E}$ così la critica, che ne fa giustamente professione, tenterà di volta in volta di avvicinarlo ad altri fenomeni, precedenti o posteriori. Si parlerà allora, con entusiasmo e una buona dose di confusione, di meraviglioso, straordinario, prodigioso, inconsueto, soprannaturale, tirando in ballo anche metafisica, realismo magico e surrealismo e spostando costantemente le frontiere del fantastico a secondo dei punti di vista e dei ravvicinamenti, giustificati o meno.

In questa notevole impresa, Stefano Lazzarin e i suoi volenterosi collaboratori si sforzano appunto di mettere un po' d'ordine nella giungla delle interpretazioni, perlomeno per quanto riguarda la letteratura fantastica italiana, forse non tanto prolifica quanto quella di certe altre tradizioni nazionali, ma che può contare al suo attivo autori non da poco, quali Landolfi, Buzzati, Capuana, Bontempelli, Savinio o Calvino per parlare solo dei più grandi. Concepito e scritto in un arco di tempo di dieci anni, il volume si presenta come un bilancio della ricezione del fantastico italiano, categoria generico-modale piuttosto che puro concetto, e fornisce uno strumento di lavoro preziosissimo, approfondito e facile da consultare, per chiunque voglia, per diletto o per lavoro, tentare di inoltrarsi nel labirinto del fantastico nostrano e delle sue interpretazioni. 
3 Il repertorio bibliografico ragionato è preceduto da uno studio di una cinquantina di pagine dovuto a Stefano Lazzarin, intitolato "Trentacinque anni di teoria e critica del fantastico italiano (dal 1980 a oggi)", dove l'autore ripercorre l'essenziale dei dibattiti sul tema, ricostruisce con cura e precisione lo sviluppo della questione e non esita a prendere posizione quando occorre per giustificare le basi del suo progetto. Pur indicando nell'Italie magique (1946) di Contini, che si voleva una "antologia del 'surrealismo italiano' degli anni Trenta e Quaranta" una pietra miliare per la percezione del fantastico, e identificando la pubblicazione de Il racconto fantastico da Tarchetti a Buzzati di Neuro Bonifazi, nel 1971, come un momento chiave per lo sviluppo del dibattito, Lazzarin mostra chiaramente come il rapporto della critica italiana al fantastico sia "per l'essenziale un dibattito post-todoroviano"1. È in effetti realmente negli anni ottanta, al seguito della traduzione e dello straordinario successo dell'Introduction à la littérature fantastique di Todorov, che si può realmente parlare di uno sviluppo della critica sul fantastico, strumento della rivalutazione di questo genere, largamente ignorato o perfino denigrato. È in quegli anni in effetti, per dirla con le parole dell'autore, che "tramonta l'ipoteca ideologica che aveva pesato sulla ricezione degli scrittori fantastici" (14), visti dalla critica vicina alle posizioni del partito comunista come semplici produttori di storielle distraenti prive di valore sociale, quanto, per conseguenza, di autentico valore letterario.

Da allora si delineano "tre modelli teorici, forgiati o riproposti fra il 1983 et il 1988, [che] risultano atti a saldarsi reciprocamente, formando un amalgama di abbagliante prestigio: il magico-surreale 'intelligente' e 'ironico' di Contini, il fantastico 'intellettuale' di Calvino, il fantastico 'colto' e mainstream di Ghidetti e Lattarulo" (31). E va altresì a svilupparsi un canone, in gran parte determinato dalle scelte di Calvino $\mathrm{e}$ dalla sua distinzione, largamente accetta, tra il fantastico 'intellettuale' e quello 'emozionale', raramente rimesso in discussione fino ad oggi.

5 Lazzarin ritorna pacatamente e con metodo sulla questione dell'indefinibilità del fantastico, "topos teorico" (38) dovuto ai paradossi affascinanti di Jorge-Luis Borges e Adolfo Bioy Casares, ma è per distanziarsene e difendere il bisogno di una ragionevole $\mathrm{e}$ indispensabile "flessibilità teorica" (43), necessaria per non perdere di vista la molteplicità degli aspetti del tema che meritano d'essere trattati. Si situa per conseguenza contro le posizioni di Manganelli (i cui ragionamenti, logici ma rigidi, impediscono fin la discussione della possibilità dell'esistenza del fantastico in quanto genere), stimando che per il fantastico - come in realtà per quanto attiene a qualsiasi altro genere letterario - l'impossibilità di definirne in maniera categorica il 'cuore' non debba peraltro voler significare che occorra semplicemente rinunciare ad ogni tentativo di definizione. Questa scelta di 'buon senso pratico' (Lazzarin spiega: "nessuno ci costringeva a murarci vivi nelle categorie todoroviane, ma neppure a seguire le orme dei teorice 'inclusivi', o a sottoscrivere ciò che ho definito il 'paradosso di Borges e Bioy Casares"'[54]) era in fondo indispensabile per un progetto volutamente enciclopedico come questo.

6 Il repertorio è organizzato in cinque sezioni: "Teorie alla prova dei testi: antologie e dintorni", "Generi, storia, tradizione", "Temi, miti, topoi", "Autori e testi", "Contesti, ricezioni, intertesti, referenti, linguaggi", e seguito da un utilissimo indice degli autori recensiti. La numerosissime recensioni - chiare, ben scritte, ricche di rinvii e di riflessioni interessanti - sono di lunghezza variabile a secondo dell'importanza del testo trattato. Con questo volume, Lazzarin e i suoi cinque collaboratori hanno messo a 
disposizione dei ricercatori e degli studenti, così come dei semplici lettori curiosi, uno strumento veramente utilissimo per il quale vanno complimentati senza riserve.

\section{NOTES}

1. P. 9, corsivo dell'autore.

\section{AUTHOR}

\section{VITTORIO FRIGERIO}

Dalhousie University 